TOMASZ KACZMAREK

ORCID: 0000-0001-6138-5280

Université de Łódź

tomasz.kaczmarek@uni.lodz.pl

\title{
LE «THÉÂTRE D’ÉVASION » DE GASTON BATY, OU COMMENT DÉPASSER LA SÉCULARITÉ
}

Le monde du drame n'est pas seulement l'univers physique auquel le corps appartient : c'est aussi et surtout l'univers spirituel qui enveloppe les âmes ${ }^{1}$.

L'expression « théâtre d'évasion »², que Gaston Baty (1885-1952) utilise pour décrire l'ensemble de ses activités, reflète ses recherches formelles étayées par des conceptions ouvertement religieuses à une époque de sécularisation où, suite entre autres à la «controverse moderniste $»^{3}$, se manifeste parfois une violente critique de la religion établie. Malgré les exactions de la Grande Guerre, le Français moyen pense à son bien-être matériel plutôt qu'à la spiritualité, ce qui l'éloigne de l'Église, et cependant les cabinets des psychiatres ne désemplissent pas ${ }^{4}$. On pourrait chercher les causes de la dépression qui touche la société industrialisée dans

${ }^{1}$ H. Gouhier, Le théâtre et l'existence, Librairie Philosophique J. Vrin, Paris 1997, p. 29.

${ }^{2}$ G. Lieber, "Gaston Baty », [dans :] Dictionnaire encyclopédique du théâtre, M. Corvin (dir.), Larousse, Paris 2001, p. 173.

${ }^{3}$ Cf. M. Guasco, Le Modernisme : Les Faits, les idées, les hommes, DDB, Paris 2007 ; É. Poulat, Histoire, dogme et critique dans la crise moderniste, Albin Michel, coll. « Bibliothèque de l'évolution de l'humanité », Paris 1996.

${ }^{4}$ J.-P. Bichet, Études sur l'anxiété dans le théâtre français contemporain, Amadée Legrand, Paris 1929. 
l'aliénation de l'individu face à la mécanisation effrénée de la vie. Emporté dans le tourbillon des techniques modernes, l'homme se trouve en porte-à-faux, car il est privé de métaphysique et dépossédé de son identité de sujet, et il a le sentiment de courir à sa perte, ses peurs ayant culminé dans l'apocalypse du premier conflit mondial. La religion institutionnalisée n'est pas à même de l'aider car elle s'avère incapable de répondre à ses aspirations fondamentales. C'est dans ce contexte que les préoccupations d'un des fondateurs du « Cartel» théâtral se concentrent dorénavant sur des questions ontologiques proches de celles des expressionnistes allemands. Ces derniers expriment une foi en l'homme qui « repose sur l'affirmation que l'esprit, partagé par tous, peut régénérer l'humanité prisonnière de la matière et lui rendre son vrai visage $»^{5}$, tandis que Baty redécouvre la « lumière divine » qui luit au fond de tout homme. Sa vision thomiste de l'univers, « qui refuse la séparation de l'âme et du corps en deux domaines radicalement disjoints, trouve en Dieu le principe qui permet de dégager une unité de la diversité du monde et de donner sens à des fragments sans cela énigmatiques $»^{6}$.

Il est intéressant à ce propos d'étudier, ne serait-ce que sommairement, quelques œuvres des dramaturges français des années 1920 et 1930, qui adhèrent à l'esthétique théâtrale et « idéologique » de Baty, sans se déclarer explicitement comme des personnes croyantes. Tout en restant méfiants envers les dogmes, symbolisés par l'Église, des écrivains tels que Henri-René Lenormand, Simon Gantillon ou Jean-Victor Pellerin constatent la perte de la transcendance qui mène inévitablement « l'homme moderne » à sa chute. Le rejet de l'enseignement de la religion institutionnalisée ne signifie pas pour autant la disparition totale du sacré dans leurs textes. Face à une civilisation de plus en plus matérialiste, les auteurs dramatiques établissent un diagnostic quelque peu pessimiste pour se mettre à la recherche de remèdes aptes à combler, comme l'aurait dit Eugène Ionesco, le « vide ontologique ». Sans aucun doute, selon ces écrivains, le drame est le lieu où se cristallisent les attentes et les déceptions religieuses qui agitent la génération tourmentée de l'entre-deux-guerres. À y regarder de plus près, les œuvres qu'ils proposent au créateur de la « Baraque de la Chimère » correspondent à un subjectivisme qui révèle une quête spirituelle.

« [...] L'odeur prend à la gorge. Le théâtre contemporain sent mauvais $»^{7}$. C'est par ces mots que Baty décrit sévèrement la condition de l'art dramatique français dans les premières décennies du $\mathrm{XX}^{\mathrm{e}}$ siècle. Profondément catholique,

${ }^{5}$ C. Mazellier-Grünbeck, Le théâtre expressionniste et le sacré, Pater Lang, coll. « Contacts », Bern 1994, p. 285.

${ }^{6}$ G. Lieber, Gaston Baty et ses auteurs : le théâtre d'évasion, thèse présentée en vue du Doctorat d'État ès Lettres et Sciences Humaines, Université Paris X-Nanterre, 1987, p. 156.

${ }^{7} \mathrm{G}$. Baty, Le masque et l'encensoir. Introduction à une esthétique du théâtre, Librairie Bloud \& Gay, Paris 1926, p. 183. 
« ayant une vision thomiste de l'univers $»^{8}$, l'auteur de Dulcinée $^{9}$ se détourne résolument des poncifs de la scène " officielle » pour ouvrir des voies modernes à une esthétique nouvelle. Dès 1921, Baty n'hésite pas à exposer ses projets de renouveau scénique dans une série d'articles, et plus tard, dans des livres programmatiques. Tout d'abord, dans Sire le Mot (1921), qui provoque une vraie controverse à l'époque, il s'en prend à l'hégémonie de la littérature au théâtre ; elle est responsable, à ses yeux, de la décadence de la représentation. Ensuite, dans La Vie de l'art théâtral des origines à nos jours (en collaboration avec René Chavance - 1952), il évoque dans l'évolution du théâtre deux courants parallèles : l'un nettement théâtral qui favorise le spectacle au détriment du drame, et l'autre qui donne de l'importance au texte, pour, à la fin, proposer « un drame intégral » ou un « théâtre total » qui fasse une synthèse des deux. Peu importe les étiquettes que l'on donne à celui qui a monté Le Dibbouk de Shalom Anski, car son rêve est de marier ces deux tendances apparemment contradictoires : c'est au metteur en scène de créer des «polyphonies vivantes » dans lesquelles le drame réunirait la littérature, la danse, la peinture et la musique. Baty praticien se veut le fervent propagateur de la rethéâtralisation, grâce à laquelle il désire mettre en scène un drame exprimant le monde dans son entier, avec « les grandes forces de la nature, le mystère, l'universelle harmonie ${ }^{10}$.

Le royaume que doit conquérir le théâtre nouveau s'étend bien au-delà, jusqu'à l'infini. Après l'homme et son mystère intérieur, après les choses et leur mystère nous touchons à des mystères plus grands. La mort, les présences invisibles, tout ce qui est par delà la vie et l'illusion du temps. Fléau des balances où s'équilibrent le bien et le mal. Ce qu'il faut de douleur pour racheter le péché et sauver la beauté du monde. Tout, jusqu'à Dieu ${ }^{11}$.

Il n'est donc pas étonnant que Baty choisisse des œuvres qui évoquent, au-delà de la réalité extérieure, une zone de mystère où se déploient sans ambages le subconscient et le surnaturel. C'est dans ce contexte qu'apparaît le terme de « théâtre d'évasion » qui, loin de signifier un simple escapisme, vise à transcender la matière pondérable en permettant ainsi de découvrir un monde spirituel non moins « matériel », ce projet étant renforcé par les conceptions religieuses manifestes du metteur en scène. Pourtant, le fondateur du « Cartel » ne s'érige point en un « dogmatiste sacerdotal ». Il est bien conscient de la sécularité de la société française, alors il doit s'adresser au public d'une autre manière. Se débarrassant de la dramaturgie conventionnelle, il rejette la vie quotidienne afin de privilégier l'essor de l'énigmatique et de l'inexplicable. Il ne renonce pas pour autant au réalisme, qui sera une sorte de tremplin pour une ouverture à l'étude de l'impénétrable. Il insiste à plusieurs reprises sur le mot « mystère », qui n'élucide rien

${ }^{8}$ J. de Jomaron, «Ils étaient quatre... », [dans :] Le théâtre en France du Moyen Âge à nos jours, J. de Jomaron (dir.), Armand Collin, coll. « La Pochothèque », Paris 1992, p. 769.

${ }^{9}$ Cette tragi-comédie, écrite par Gaston Baty, a été publiée en 1938.

${ }^{10}$ Propos de Baty dans Bulletin de la Chimère 1, février 1922, p. 3.

${ }^{11}$ G. Baty, Rideau baissé, Bordas, Lyon 1949, p. 217. 
(car ceci suggérerait une visée didactique), mais qui constitue une interrogation « devant les grandes instances de l'existence humaine ${ }^{12}$. Dès lors, tout en renouant avec le théâtre religieux qu'il adapte pour la scène contemporaine, Baty a l'ambition d'instaurer un "mystère moderne ", qui tienne compte des malheurs de l'homme de la première moitié du $\mathrm{XX}^{\mathrm{e}}$ siècle.

Parmi les auteurs qui se rallient avec enthousiasme à l'esthétique de Baty, figure Henri-René Lenormand (1882-1951). Cet athée invétéré, grand admirateur de Friedrich Nietzsche, de Fiodor Dostoïevski et avant tout d'August Strindberg, jouit d'une renommée d'écrivain amoral, dont on donnera à l'œuvre l'étiquette de « théâtre de l'inquiétude ». De fait, dans une majorité écrasante de ses drames, Lenormand dépeint nombre de personnages souffrants qui cherchent éperdument un apaisement à leur angoisse existentielle, toutes leurs tentatives de remédier à leur condition précaire étant tout de même vouées à l'échec. Ainsi, dans Les Ratés (1921), drame que Baty monte au Théâtre Montparnasse en $1937^{13}$, nous assistons à la lente dégradation morale et physique d'un couple, désigné par les simples pronoms «Lui » et « Elle », comme si le dramaturge voulait faire d'eux, à l'instar des expressionnistes, les incarnations symboliques de l'humanité déchue. L'homme est un mauvais écrivain en mal de gloire, tandis que la femme, rongée par des ambitions artistiques illusoires, doit se contenter d'être une actrice de troisième ordre. Ils se mettent en route pour accompagner des cabotins qui jouent dans des salles insalubres. Ces clochards jetés dans le monde contre leur gré se meuvent comme des silhouettes aux contours flous, comme s'ils étaient délaissés de Dieu. Au départ, ils crient encore leur dégoût, protestent contre leur malheur, mais plus ils avancent, plus ils abandonnent l'espoir. Privés de tous points de repère, ils ne connaissent que la misère et la douleur, et dès lors, ne voyant aucune voie de secours, ils s'engouffrent dans la déchéance tout en précipitant leur destin fatal. Ils se courbent, se couvrent de boue avec une joie délirante : l'homme force sa compagne à se prostituer afin de subvenir à leurs besoins matériels, mais, ne supportant pas l'infidélité de la femme, il la tue avant de mettre fin à ses jours. Lenormand présente la tragédie moderne de l'humanité condamnée à sa perte, le fatum qui pèse sur elle :

C'est 1'effondrement inéluctable non seulement des aspirations spirituelles et des ambitions, mais aussi de toute tendresse et de toute quiétude. C'est l'enlisement progressif et inconjurable dans l'avilissement moral, dans la bassesse, dans la boue humaine jusqu'au meurtre et au suicide en une chambre d'hôtel anonyme et banale ${ }^{14}$.

Si le chemin de ces hères semble ne pas dépasser le cadre réaliste, il n'est pas sans rappeler le calvaire spirituel des protagonistes expressionnistes. Or, malgré

12 G. Lieber, Gaston Baty et ses auteurs..., p. 157.

13 Georges Pitoëff met en scène la pièce de Lenormand en 1920 au Théâtre des Arts de Genève.

14 P. Blanchart, «Le théâtre de Henri-René Lenormand. Apocalypse d'une société », Masques, Paris 1947, p. 36. 
ses contours naturalistes, la pièce « est toute intérieure $»^{15}$, car le vrai drame se déroule dans les âmes tourmentées des pauvres vagabonds. Il serait légitime d'y voir un Stationendrama allemand, dont la forme présente la destinée humaine « comme un cheminement parsemé d'épreuves que le héros doit affronter au cours de son évolution ${ }^{16}$. De fait, la tournée ne signifie pas uniquement un déplacement physique, elle constitue un prétexte pour exposer une vraie via crucis de l'homme moderne. Nous connaissons le monde par le biais du psychisme du souffrant, ce qui revient à dire que l'espace évoqué par le dramaturge est une «projection du Moi des personnages ${ }^{17}$.

Baty s'apprête encore à jouer les deux «pièces climatiques » de Lenormand (Le Simoun - $1920^{18}$, À l'Ombre du mal - 1925), dans lesquelles le dramaturge repropose la chute de l'homme « raisonnable » qui, sous l'emprise du climat tropical et des sorcelleries des Africains, s'enlise dans la débauche et finit par succomber face aux forces de la nature, mais c'est L'Amour magicien, qu'il monte au Studio des Champs-Élysées (1929), qui retient une attention plus particulière du metteur en scène. Dans cette œuvre «mystique » qui vaudra à son auteur le qualificatif $d$ ' « halluciné », les personnages se déplacent constamment à la frontière du réel et du surnaturel. Le dramaturge, inspiré par La Survivance de l'âme et son évolution après la mort (1920) de Pierre Émile Cornillier, y raconte l'histoire d'une femme possédée par une morte. L'action du drame peut se résumer en quelques mots : Albert Carolles, inconsolable après la disparition mystérieuse de sa femme (on présume qu'elle s'est noyée), vit avec une petite secrétaire irlandaise qu'il exhorte, par l'intermédiaire d'un hypnotiseur, à entrer en contact avec sa bien-aimée défunte. Mais voici que la morte revit à travers le corps de la jeune fille : elle reproche à Albert sa mauvaise conduite. Ses mains invisibles étranglent l'imposteur, mettant fin à la vie du couple. La fin de l'action rappelle les excès du Grand-Guignol, mais Lenormand ne croit pas aux forces surnaturelles, et encore moins à la survivance de l'âme. Les séances médiumniques lui servent à étudier la psychologie de son protagoniste et si les revenants apparaissent, ils ne sont que des matérialisations de son psychisme fragile. Le dramaturge essaye donc dans ce texte de se livrer à une analyse minutieuse de l'âme détraquée, mais cette approche quasi psychiatrique qui cherche à tout expliquer n'est pas bien accueillie par la critique. Même la mise en scène magistrale de Baty, avec ses effets visuels (de clairs-obscurs « à la Rembrandt ») et acoustiques ainsi que l'excellente interprétation du rôle du médium par Marguerite Jamois, ne garantissent pas une longue vie à cette pièce. Tout porte à croire que l'« esprit cartésien " propre aux

15 P. Audiat, « Les Ratés », Paris-soir, 7 avril 1937.

${ }^{16}$ C. Mazellier-Grünbeck, op. cit., p. 65.

17 W. Steffens, «Théâtre », [dans :] L'expressionnisme, L. Richerd (dir.), Somogy, Paris 1993, p. 159.

18 Baty monte à nouveau le texte de Lenormand en 1930 au Théâtre Pigalle. 
écrivains français semble ne pas leur permettre de s'abstenir de tentatives d'explication rationaliste des phénomènes mystérieux.

Baty monte deux pièces qui abordent un sujet analogue : en 1926, Le Dibbouk d'Anski, et quatre ans plus tard Bifur, de Simon Gantillon (1887-1961). La première, basée sur une légende hassidique, présente la possession de Léa par l'âme désespérée de son défunt amant Hanan, qui est condamnée à voler entre le monde des vivants et celui des morts, tandis que la deuxième met en scène une femme dont l'âme sera ressuscitée dans le corps d'une autre. Si le drame d'Anski remporte un franc succès, le texte de l'auteur français partage le même sort que L'Amour magicien : n'ayant pas enthousiasmé le public, la pièce ne reste pas longtemps à l'affiche. Lenormand comprend les raisons de l'échec de son œuvre (ce qui est aussi valable pour celle de Gantillon) et ne tarde pas à avouer que Le Dibbouk a captivé les spectateurs parce qu' « ils n'étaient, par l'auteur, ni mis en discussion ni justifiés du point de vue de la psychiatrie ${ }^{19}$. Quand Gantillon débute en tant que dramaturge avec Cyclone, monté par Baty à la Baraque de la Chimère en 1923, il tente de décrire la décomposition psychique des marins sous l'influence du climat destructeur. Mais son faible pour le réalisme fait de la pièce une simple histoire de matelots aux prises avec la mer agitée, laissant de côté sa dimension eschatologique. Ce n'est qu'avec Maya (1924), où le dialogue comporte « une part d'indétermination $»^{20}$, qu'il s'impose comme un écrivain novateur.

Dans cette pièce qui, selon certains, est « un poème à la gloire de la prostituée $»^{21}$, le dramaturge arrive à percer l'écorce extérieure de la réalité pour donner libre cours à l'étude des profondeurs imperceptibles de l'âme humaine. De prime abord Baty ne tombe pas sous le charme du texte, mais, après réflexion, il l'accepte sous réserve de quelques retouches de surface. Il ne regrettera pas sa décision, car l'œuvre de Gantillon (qui connaîtra un succès international et sera même portée au grand écran) lui permet de réaliser sur scène son idéal d'un " drame intégral » où le réalisme se répand en se fondant harmonieusement dans le symbolisme. De fait, au niveau de l'intrigue, l'auteur de Marine relate tout simplement la vie des femmes de mœurs légères qui vendent leur corps dans le port de Marseille, mais il renonce délibérément aux vulgarités de leur existence afin d'en accentuer la dimension poétique. Dans le sillage du théâtre d'évasion, l'écrivain campe ses pauvres créatures déchirées entre les désirs corporels et spirituels. Loin de porter un jugement moralisateur, il ne les présente pas comme des filles ordinaires ou grossières, sexuellement insatisfaites, mais comme des personnes malheureuses, incapables de s'affranchir de leur vice dégradant. Baty voit dans ces demi-mondaines moralement écorchées des candidates à la sainteté. De plus, malgré les relents réalistes de la pièce, les amazones de Gantillon ne semblent pas provenir

${ }^{19}$ H.-R. Lenormand, Les Confessions d'un auteur dramatique, t. 2, Albin Michel, Paris 1953, p. 137.

${ }^{20}$ M. Vaïs, L'écrivain scénique, Presses de l'Université du Québec, Montréal 1978, p. 44.

${ }^{21}$ M. Raymond, Le Jeu retrouvé, Éditions de l'Arbre, Montréal 1943, p. 86. 
de ce monde matériel et - en particulier Bella, la propriétaire de la maison de passe - elles rappellent plus des êtres éthérés que des êtres en chair et en os. Elles tournent, tels des esprits dérangés, autour de la maîtresse de la maison close, comme si elles reflétaient les différentes instances du psychisme de leur patronne. Il est vrai que Gantillon présente une situation réelle, une sorte de « tranche de vie ", mais il incite toujours à scruter ce qui se passe derrière l'écran souvent mensonger de la réalité. Dans cette perspective, nous ne devons pas percevoir Bella dans sa dimension physique mais tenter de découvrir sa nature mystérieuse. Il n'est pas étonnant que l'écrivain intitule sa pièce Maya, titre qui fait ouvertement allusion à la philosophie hindoue. Bella-Maya se présente comme une illusion du monde physique, que notre conscience considère comme la seule réalité existante : elle empêche les mortels de se saisir de la vérité transcendante. Dans un premier temps, l'héroïne se montre comme une divinité qui porte un voile trompeur, mais dans un deuxième temps, elle ouvre la voie aux hommes vers l'au-delà de la vie temporelle douloureuse, pour trouver l'apaisement de leurs maux. C'est elle qui console les pauvres clients qui, à vrai dire, ne cherchent pas tant les plaisirs charnels que les mots de réconfort. C'est Bella-Maya qui vient en aide aux solitaires et aux mal-aimés avides d'une foi apte à régénérer leur réalité matérielle. Ainsi, en renouant avec la sagesse orientale, Baty fait de la figure de la prostituée une vraie déesse susceptible de sauver l'humanité en perte de valeurs, tout le spectacle s'offrant au public comme un " rite moderne ». La succession de tableaux juxtaposés, où le symbolisme empiète constamment sur le réalisme, concourt à créer une atmosphère quelque peu énigmatique. Sous cet angle, la construction du drame, où l'action au sens dramatique est presque inexistante, témoigne de la mise en valeur du monde invisible. Les didascalies de Gantillon, qui agrémentent son texte d'éléments visuels, préparent incontestablement le terrain au rituel. Le metteur en scène invite les spectateurs à participer au cérémonial de l'« élévation » ${ }^{22}$ de l'homme. Pour le concrétiser scéniquement, Baty s'appuie sur le son, les éclairages, les accessoires et d'autres moyens théâtraux qui permettent de donner un spectacle intégral, censé retrouver la force des anciens mystères religieux. Rejetant la représentation matérielle, il ne construit pas de plafond, de portes ou de fenêtres, mais propose des cadres suggérant leur existence. Ses toiles, dont la perspective conventionnelle est résolument renversée, conduisent à une vision symbolique ou abstraite plutôt qu'à une simple description. Le théâtre de Gantillon reçoit ainsi une consécration, car Baty rétablit ouvertement les liens brisés avec l'origine sacrée du drame, la scène devenant un lieu de communion.

Quant à Jean-Victor Pellerin (1889-1970), il semble se situer dans le sillage de la pensée philosophique de Søren Kierkegaard, qui sera reprise par Karl Jaspers et Gabriel Marcel (ce dernier, au demeurant, figure parmi les membres

22 J.D. Delaney, The Element of Totality in the Theater of Gaston Baty, The University of Arizona, Departement of Romance Languages, Tucson 1968, p. 177. 
du comité de lecture constitué par Baty), en ce qu'elle dénonce l'immersion moderne de l'individu dans la foule. En qualité d' « existentialiste autodidacte », le dramaturge met en garde, tel l'auteur du Journal Métaphysique, contre « cette acception passive de consignes qui tendent à ne plus faire de [l'être humain] qu'un simple rouage de machine, et il le rappelle à son existence authentique $»^{23}$. Déjà dans sa première œuvre dramatique, Intimitée ${ }^{24}$ (1921), qui connaîtra un succès éclatant, Pellerin s'intéresse à la vie « intérieure » de ses personnages aux prises à une existence morne et privée de sens. C'est en révélant les désirs refoulés de ce couple bourgeois que le dramaturge arrive à dévoiler leur solitude existentielle. Pourtant, tels des fantoches dépourvus de toute initiative, ils ne sont pas capables de changer leur condition précaire, et plutôt que de se révolter, ils se résignent en se permettant de temps à autre de s'abandonner à leurs rêves, ultimes havres de paix illusoire. Pour matérialiser la déréliction des personnages, Baty divise l'aire de jeu en deux zones : la première présente un décor réaliste symbolisant la vie monotone des solitaires, tandis que la deuxième, tendue de velours noir, devient un terrain où se « concrétisent » leurs rêves d'antan.

Dans Têtes de rechange ${ }^{25}$ (1926), le deuxième drame que Baty met en scène au Studio des Champs-Élysées, l'écrivain décrit l'histoire d'un jeune brasseur d'affaires au nom inhabituel, Ixe, qui, lassé d'une vie monotone et insupportable, tente de s'évader dans un monde féerique où il peut s'adonner corps et âme aux plaisirs que les «contraintes bourgeoises » lui interdisent. Cependant, comme dans la pièce précédente, le protagoniste fuit le monde mécanisé et impersonnel sans trouver la sérénité durable tant désirée. Il est vrai qu'il se met en route à la recherche de l'espoir, mais au cours de son itinérance, son identité, au lieu de se raffermir, s'éparpille en plusieurs « moi » aussi anonymes que son prénom. L'atomisation du businessman prouve qu'il se dilue dans le magma de la banalité quotidienne, le condamnant à mener une existence inauthentique. En montant cette pièce, Baty n'a pas seulement recours à un jeu de toiles créant une atmosphère prenante, celle d'un monde déshumanisé, mais adopte aussi certaines techniques modernistes de jeu corporel : il fait des acteurs des silhouettes-objets qui s'acharnent sur le protagoniste. Le metteur en scène se plaît à jouer cette œuvre, car elle exemplifie la tentative de métamorphose intérieure du personnage principal ; il l'aime tout autant pour ce « va et vient » entre le monde réel et onirique, ce qui lui permet de concevoir le décor simultané, mais cette « suite de sketchs ", malgré sa critique véhémente de la société industrialisée, rappelle plutôt une revue de music-hall. Le grotesque prime sur le sérieux du sujet abordé. Ce n'est que

${ }^{23}$ R. Campbell, L'Existentialisme, Foucher, Paris 1969, p. 33.

${ }^{24}$ Cette pièce est représentée pour la première fois par les Compagnons de la Chimère au Théâtre des Mathurins le 9 mai 1922. Elle sera reprise à la Baraque de la Chimère en 1923, puis au Studio des Champs-Élysées en 1925, toujours dans une mise en scène de Gaston Baty.

${ }^{25}$ Cette pièce sera reprise par Jean Le Poulain en 1964 au Théâtre des Bouffes-Parisiens. 
dans Cris des cœurs (1928) et surtout dans Terrain vague (1931) que la plume de Pellerin se fait un peu plus grave.

Bouleversé par la mort prématurée de son fils unique, le dramaturge cherche dans son écriture à apaiser sa douleur. Dès lors, il reprend le motif de l'évasion du monde matériel, qui, cette fois, ne se réduit plus à un escapisme frivole, mais prend la forme d'une quête spirituelle de la transcendance. En effet, dans la dernière partie de Cris des coeurs, drame monté par Baty au Théâtre Montparnasse (1936), nous voyons un jeune sculpteur qui ne pense point aux privilèges et à la célébrité que son art original pourrait lui rapporter. Animé par une force bienfaisante, incompris de ses « amis » préoccupés uniquement par les questions matérielles, le jeune artiste trouve son génie en Dieu, car il crée avec le cœur. Sa foi sincère n'est pas celle des bigots, ni même des théologiens, elle se manifeste à travers l'expression de l'impossible : il désire créer « quelque chose de grand et de simple à la fois, de supérieur à tout et d'accessible à tous, quelque chose qui, bien que dominant la vie, resterait vivant,- - une sorte de dieu humain, quoi $! »^{26}$. Rechercher Dieu, c'est trouver les vérités masquées par les apparences, c'est aussi la prise de conscience de l' « étincelle divine » qui « habite la poitrine de l'homme $»^{27}$. Pellerin incorpore à la fin de son drame une vision parfaitement métaphysique quelque peu pompeuse, mais c'est dans cette scène qu'il renoue avec les mystères médiévaux tout en les «modernisant». Au moment où le jeune sculpteur retrouve la paix dans la solitude, on entend les voix lointaines des Errants et un garçon se fait voir. Un enfant mort apparaît, débordant de vie et de santé, et invite son père et sa mère à le rejoindre par la prière. Et l'artiste de se mettre à son ouvrage avec hardiesse. C'est ainsi que le dramaturge illustre l'un de ses thèmes privilégiés, celui de la «transfiguration et de la rédemption par l'amour du divin $»^{28}$.

Terrain vague, monté par Baty au Théâtre Montparnasse (1931), n'a pas été apprécié par la critique. La pièce relate l'évolution intérieure d'un homme d'affaires, André Lefèvre, qui, fatigué de son existence mécanique, se révolte pour retrouver la plénitude dans l'amour. Pellerin décrit l'asservissement de l'homme à la machine qui décide pour lui. La première partie est justement focalisée sur la déshumanisation qui provoque l'aliénation de l'individu, perdu dans une masse déraisonnable composée de robots plutôt que d'êtres humains. C'est dans la deuxième partie seulement que nous assistons à la métamorphose du protagoniste qui finit par s'insurger contre le système inepte dans lequel il vit. Nous le voyons au théâtre où, de manière mystérieuse, sa vie intime est jouée sur scène. Profondément perturbé, André pousse un cri étouffé avant de quitter sa place et de monter sur le plateau. Sur ces entrefaites, face au public, son histoire prend la forme d'un cheminement parsemé d'épreuves qu'il doit affronter au cours de son évolution.

\footnotetext{
26 J.-V. Pellerin, « Cris des cœurs », Masques, Paris 1928, p. 120.

27 C. Mazellier-Grünbeck, op. cit., p. 244.

28 A. Simon, Gaston Baty : théoricien du théâtre, Klincksieck, Paris 1972, p. 68.
} 
Il rencontre des personnages bizarres (qui en fait incarnent les instances de son psychisme inconscient) avec lesquels il s'entretient, ahuri de leur stupidité et de leur atrocité. À ce moment, Baty présente des personnages déformés par un éclairage qui souligne leur aspect aussi grotesque qu'effrayant (par exemple un énorme individu fumant un cigare gigantesque et portant d'immenses lunettes). L'homme d'affaires croise aussi un petit garçon qui, à son grand étonnement, s'avère être lui-même quand il était enfant. C'est grâce à lui qu'André prend conscience du temps qui s'écoule impitoyablement, et donc, de sa vie ratée. Le petit lui suggère de rechercher l'innocence de l'enfant, de retrouver un bonheur sincère dans l'amour. André est comme éclairé. Il rejette le mercantilisme auquel il a sacrifié sa joie de vivre. Il sort du théâtre troublé, mais décidé à changer son existence triste et maussade. La troisième partie débouche sur une scène fantasmagorique : avec son épouse, le protagoniste s'envole heureux dans le ciel. Dorénavant le couple, délivré de toute attache à la matérialité dégradante, vit des moments de vie intense, renforcés par une immense lumière (magistralement maniée par Baty) dont l'intensité s'accroît en fonction de la sérénité grandissante.

Pellerin semble donner ainsi à son personnage le statut de nomade, car il est un continuel voyageur, un Homo Viator dont la quête se manifeste soit comme un itinéraire externe au cours duquel le protagoniste se met en marche en découvrant le questionnement qui l'agite, soit comme une descente interne, une sorte de vivisection intérieure de l'âme tourmentée, l'une n'excluant pas l'autre. Les pérégrinations d'André ne font pas penser au stade esthétique de Kierkegaard, car l'errance de cet ancien employé n'est pas sans orientation. Au contraire, elle vise, comme l'aurait dit Marcel, à ce que l'homme atteigne à son existence authentique et « réelle » qui, grâce à l'amour et loin d' ' étouffer » le désespoir, lui assure la liberté et, en fin de compte, l'espérance.

Gaston Baty est passé à la postériorité comme un créateur fort original. De fait, il a tourné le dos au règne sans partage du Théâtre de Boulevard (qu'il abhorrait sincèrement) tout en proposant une panoplie de procédés censés renouveler la scène française. Et ses objectifs étaient clairement de nature religieuse, ce qu'il n'a pas manqué d'affirmer à maintes reprises : " en réaction contre la tradition française, toute cartésienne et janséniste, j'essaie de servir un théâtre selon Saint Thomas $"{ }^{29}$. Néanmoins, il ne faut pas confondre la foi religieuse de ce grand homme de théâtre avec ses convictions professionnelles d'esthète de la scène. S'il tient à monter des pièces abordant des questions eschatologiques, il ne se fait pas pour autant le propagateur du catéchisme catholique. "À tous les stades de sa carrière, il manifeste, à l'égard de la religion, comme de la politique,

${ }^{29}$ F. Porché, « Gaston Baty », Revue de Paris, 15 août 1935, p. 992. 
une indépendance absolue : il juge, choisit, et pense uniquement en fonction du théâtre $»^{30}$. Loin de s'inscrire sous l'égide de la théologie, Baty crée son propre mysticisme : la scène est pour lui un tremplin à rêve, qui vise à éveiller des « échos lointains » dans l'âme du spectateur, le théâtre étant considéré comme " un refuge et un lieu de ressourcement $»^{31}$. Faut-il ajouter que pour ce visionnaire, le texte n'est pas tout : c'est au « créateur scénique » de dévoiler ce qui se cache derrière les paroles :

Le poète a rêvé une pièce. Il met sur le papier ce qui en est réductible aux mots. Mais ils ne peuvent exprimer qu'une partie de son rêve. Le reste n'est pas dans le manuscrit. C'est au metteur en scène qu'il appartiendra de restituer à l'œuvre du poète ce qui s'en était perdu dans le chemin du rêve au manuscrit ${ }^{32}$.

Malgré l'hétérogénéité des œuvres que Baty choisit pour son théâtre, on peut leur trouver un dénominateur commun : la dimension religieuse, qui se traduit avant tout par la quête spirituelle de l'homme moderne face à la déshumanisation de la société industrialisée. Cette recherche prend souvent la forme d'un chemin au cours duquel on assiste à la métamorphose intérieure de l'individu en conflit avec ce qui l'entoure. Il est pourtant rare que s'opère une vraie « conversion » du personnage. Les Ratés de Lenormand sont un exemple de " drame à stations ", qui permet à l'écrivain de montrer comment, étape par étape, les protagonistes s'enlisent petit à petit dans la déchéance avant de se tuer. Le dramaturge décrit ainsi la condition précaire de l'homme privé de tous points de repère, tout en mettant l'accent sur la vie inconsciente de ses clochards. En dépit d'une prolifération verbale parfois excessive, l'auteur de la Mixture réussit à représenter un « espace intérieur » et à écarter la réalité extérieure, ce que Baty retranscrit plastiquement dans ses mises en scène. Le réalisme discret et le subjectivisme sont aussi au rendez-vous dans la production dramaturgique de Gantillon où l'itinérance s'accomplit au travers de l'analyse intérieure de l'âme souffrante. Les personnages s'évadent du réel pour plonger dans le monde invisible de leur psychisme perturbé. Tant Lenormand que Gantillon proposent des pièces qui donnent « à sentir plutôt qu'à comprendre, à rêver plutôt qu'à raisonner $»^{33}$, mais si le premier est profondément pessimiste, le deuxième campe sur ses positions de mélancolique désabusé. Contrairement à ses confrères, Pellerin présente une recherche spirituelle qui ne se solde pas par un échec. Cris des cœurs et Terrain vague ont pour toile de fond l'idée de rupture entre les hommes aliénés dans un monde hostile. Le sculpteur et le businessman réussissent, au bout de leur itinéraire, à dépasser la morosité par l'amour et la foi. Aimer Dieu ne signifie pourtant pas se soumettre aux interdits ecclésiastiques, mais professer l'amour du prochain. Le dramaturge

${ }^{30}$ P. Blanchart, Gaston Baty, coll. « Choses et gens de théâtre », Éd. Nouvelle Revue Critique, Paris 1939, p. 63.

${ }^{31}$ G. Lieber, « Gaston Baty », p. 173.

${ }^{32}$ G. Baty, Rideau baissé, p. 219.

33 G. Lieber, « Gaston Baty », p. 166. 
est loin d'ôter à l'homme son anxiété (ce qu'il a de meilleur) et tout en affirmant que Dieu est le fondement de l'être, la quête du divin se fait à travers un cheminement douloureux, qui passe par l'acceptation de la souffrance. En effet, d'après Jacqueline de Jomaron, ces œuvres permettent à Baty d'introduire en France un style scénique proche de l'expressionnisme. "Le metteur en scène joue sur la stylisation du décor, les clairs-obscurs, les alternances de faisceaux lumineux, les projections d'ombres sur écran qui dédoublent ou détriplent les personnages $»^{34}$.

Quoi qu'il en soit, tout en affirmant incontestablement l'importance de la mise en scène, Baty a concouru au développement d'une nouvelle dramaturgie en lançant le théâtre du subconscient de Lenormand, l'esthétique d'évasion de Gantillon et les extravagances fantaisistes de Pellerin. Le metteur en scène désire toujours rethéâtraliser le théâtre et pour ce faire, il veut le purifier du réalisme au profit du surnaturel et du rêve. Avant de consacrer ses dernières années aux marionnettes, il constate avec une conviction inébranlable :

Un théâtre nouveau va grandir. Déjà voici sur la montagne les pas des messagers. Il ne recommencera pas plus le théâtre médiéval que celui-ci ne recommençait le théâtre grec, mais il vivra des principes qui les ont fait vivre : il sera «thomiste » comme eux. Eschyle et Sophocle, qui ne prévoyaient ni le dogme, ni la morale des Évangiles, ont œuvré selon l'esthétique catholique. Peut-être les ouvriers du renouveau théâtral ne seront-ils pas moins étrangers à l'Église ; c'est à l'esthétique catholique qu'ils obéiront eux aussi, parce que toute autre mutile, appauvrit et dessèche le drame. Par delà la renaissance et la réforme se renoue la tradition française ${ }^{35}$.

\title{
THE “ESCAPE THEATRE” OF GASTON BATY OR HOW TO OVERCOME SECULARITY
}

\author{
Summary
}

In this article, we study the works of French playwrights from the 1920s and 1930s who adhere to Gaston Baty's theatrical aesthetics, without explicitly declaring themselves as believers. While remaining wary of dogmas symbolized by the Church, writers such as Henri-René Lenormand, Simon Gantillon and Jean-Victor Pellerin note the loss of transcendence that inevitably leads "modern man" to his downfall. The rejection of the teaching of institutionalized religion does not mean a total disappearance of the sacred in their texts. In the face of demonic civilization, dramatic authors make a somewhat pessimistic diagnosis in search of remedies likely to fill the "ontological vacuum." Undoubtedly, according to these writers, drama is the place where expectations and religious disappointments that agitate this tormented generation of the interwar period crystallize.

Key words: Gaston Baty, Henri-René Lenormand, Simon Gantillon, Jean-Victor Pellerin, escape, secularity, spirituality.

\footnotetext{
${ }^{34}$ J. de Jomaron, op. cit., p. 770.

35 G. Baty, Le masque et l'encensoir..., p. 183.
} 